\title{
LITHIUM IN GIANTS
}

\author{
SUCHITRA BALACHANDRAN \\ Astronomy Department, The Ohio State University, \\ 174 West 18th Avenue, Columbus OH 43202, USA
}

In the standard stellar model, lithium burning does not occur during the first-ascent giant branch phase. The surface lithium abundance in a first-ascent or clump giant is thus the product of the lithium abundance of its main sequence progenitor and subsequent dilution by the deepening convective envelope. Iben's $(1967 \mathrm{a}, \mathrm{b})$ early calculations predicted that the dilution factor would vary between a factor of about 28 in a $1 \mathrm{M}_{\odot}$ star to a factor of 60 in a $5 \mathrm{M}_{\odot}$ star. More recent calculations with improved physics show remarkably little change in these predicted dilution factors (Pinsonneault, pvt. comm.). Since Li only survives in the outer $2-3 \%$ by mass of a main sequence star, the maximum redgiant abundance is predicted to be $\sim$ $\log \epsilon(\mathrm{Li})=1.5$ to within a factor of two. Even lower abundances will result if surface $L i$ is destroyed during the main sequence phase. Lithium depletion on the main sequence has been well documented in stars of spectral type mid-F and later and have been explained by a variety of non-standard mechanisms (e.g., rotation, diffusion and turbulence). Mixing induced by such mechanisms is not expected in high-mass stars $\left(\mathrm{M}_{\odot} \geq 1.5\right)$ which have insignificant surface convection. Yet recent observations reveal that non-standard processes may be at work in these stars as well.

Lithium abundances far below the predicted maximum value are seen in high-mass cluster giants, and in some clusters large spreads in $\mathrm{Li}$ are observed in giants of roughly the same mass (Gilroy 1989). If, in concordance with the standard-model prediction, one assumes that $\mathrm{Li}$ burning on the giant branch is unlikely, then both the destruction and the scatter of $\mathrm{Li}$ must be accounted for on the main sequence. Since the surface convection zones are insignificant, other forms of Li-loss (e.g. mass loss, meridional circulation) may have to be resorted to. Unfortunately the main sequence progenitors of most of these giants are too hot to have a measurable $\mathrm{Li}$ I feature, but observations of field late-A stars by Burkhart and Coupry (1991) reveal some evidence for surface Li-loss. Follow-up observations of 
$\mathrm{Li}$ in clusters, and $\mathrm{Be}$ and $\mathrm{B}$ abundance determinations would be useful. Lithium burning on the giant branch should be investigated theoretically.

Even more puzzling are the dozen or so giants with $\mathrm{Li}$ abundances larger than the predicted maximum value. Some like HD 112127 (Wallerstein and Sneden 1982) have $\mathrm{Li}$ abundances of $\log \epsilon(\mathrm{Li})=2.7$, only a factor of 4 lower than the meteoritic value. Understanding these large Li abundances presents a challenge in the face of the low ${ }^{12} \mathrm{C} /{ }^{13} \mathrm{C}$ isotope ratios (indicative of normal giant-branch mixing) measured in at least a few of these giants. For the observed $\mathrm{Li}$ to be a main sequence remnant, a substantial concentration of Li must have occurred during the pre-main sequence phase before the stellar interior is hot enough to burn $\mathrm{Li}$. Since anomalously $\mathrm{Li}$ rich dwarfs have yet to be observed, this concentration must be so subtle that it remains unobservable (hidden below the surface convective zone) during the main sequence phase. An equally challenging alternative from the theoretical viewpoint is that $\mathrm{Li}$ is manufactured during the first ascent giant phase. The manufacture of $\mathrm{Li}$ has been predicted and observed during the asymptotic giant branch phase, but is not predicted to occur during the first ascent phase. The possible production of Li during the first ascent was suggested by Fekel and Balachandran (1993) to account for their finding that a number of the single Li-rich giants were chromospherically active; both angular momentum and $\mathrm{Li}$ were hypothesised to be dredged up from the stellar interior. Since the diffusive properties of Be and B mimic that of $\mathrm{Li}$, the abundances of these elements can be used to uncover the cause of the Li-rich giants.

The vast majority of field giants have $\mathrm{Li}$ abundances well below the predicted maximum (Brown et al. 1989) and are consistent with having evolved from low-mass stars which have undergone depletion on the main sequence. The peculiar giants are intriguing and understanding them will be challenging and potentially very useful.

\section{References}

Brown, J. A., Sneden, C., Lambert, D. L., and Dutchover, E. 1989, ApJS, 71, 293.

Burkhart, C. and Coupry, M. F. (1991), $A \& A, 249,205$.

Fekel, F. C. and Balachandran, S. (1993), $A p J, 403,708$.

Gilroy, K. K. (1989), Ap $J, \mathbf{3 4 7}, 835$.

Iben, I., (1967a), $A p J, 147,624$

Iben, I., (1967b), $A p J, 147,650$

Wallerstein, G. and Sneden, C. (1982), $A p J, 255,577$. 